\title{
Primary intra-osseous Hybrid Schwannoma-Perineurioma in the mandible
}

\author{
Mariana-Lobo Bergamini ${ }^{1}$, Luanna-Priscilla-Montenegro Noberto ${ }^{2}$, Gabriel-Barroso-Marocco-de Abreu Torres ${ }^{2}$, \\ Haroldo-Arid Soares ${ }^{2}$, Fabiana Martins ${ }^{3}$, Andre-Luiz-Ferreira Costa ${ }^{4}$, Suzana-Orsini-Cantanhede-Machado de \\ Souza ${ }^{1}$, Paulo-Henrique Braz-Silva ${ }^{1,5}$
}

\author{
${ }^{1}$ Department of Stomatology, School of Dentistry, University of São Paulo, São Paulo, Brazil \\ ${ }^{2}$ Division of Odontology, Municipal Hospital Carmino Caricchio, São Paulo, Brazil \\ ${ }^{3}$ Department of Dentistry, University of Santo Amaro, São Paulo, Brazil \\ ${ }^{4}$ Postgraduate Program in Dentistry, Cruzeiro do Sul University, São Paulo, Brazil \\ ${ }^{5}$ Laboratory of Virology, Institute of Tropical Medicine of São Paulo, School of Medicine, University of São Paulo, São Paulo, \\ Brazil
}

\section{Correspondence:}

Department of Stomatology

Division of General Pathology

School of Dentistry, University of Sao Paulo

Sao Paulo, SP Brazil

Av. Prof. Lineu Prestes

2227 Cidade Universitária São Paulo-SP Brazil

pbraz@usp.br

Bergamini ML, Noberto LPM, Torres GBMA, Soares HA, Martins F, Costa ALF, de Souza SOCM, Braz-Silva PH. Primary intra-osseous Hybrid Schwannoma-Perineurioma in the mandible. J Clin Exp Dent. 2020;12(9):e888-91.

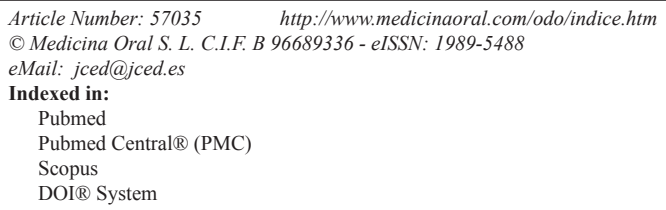

\begin{abstract}
Benign nerve sheath tumours include perineuriomas, schwannomas and neurofibromas. Hybrid schwannoma-perineurioma represents a cutaneous, subcutaneous or occasionally intra-osseous tumour with schwannian cytomorphology and perineurioma-like architecture consisting of a mixture of both types of cells. These tumours can develop at any age and there is no gender-predilection. Tongue is the most frequently affected site, followed by palate, mouth floor, jugal mucosa, lips and, more rarely, mandible. We present a case of hybrid tumour with schwannoma-perineurioma morphology located on the right mandibular body (intra-osseous) of a 54-year-old female patient. The tumour was symptomatic and evolving for six months. Microscopically, it was encapsulated and highly cellularised, presenting fascicular aspect and exhibiting scant mitotic activity. The tumour consisted of distinct cellular populations involving fusiform cells, cells with wavy and hyperchromatic nucleus or even epithelioid cells. Positive immunostaining for S-100 and epithelial membrane antigen (EMA) was observed. The lesion was completely removed under general anaesthesia, with the patient showing no clinical or radiographic sign of relapse after twoyear follow-up. Despite the limited knowledge on the pathogenesis of Hybrid Schwannoma-Perineurioma, these tumours seem to present a non-aggressive biological behaviour. Conservative surgery provides adequate solution without recurrence, even after a long-term follow-up.
\end{abstract}

Key words: Nerve sheath tumours, Schwannoma, Perineurioma, Immunohistochemistry. 


\section{Introduction}

Hybrid tumours of peripheral nerve sheath are benign mixed neoplasias with characteristics of more than one type of nerve sheath, encompassing a spectrum of well-defined clinical-pathological entities, such as schwannomas, neurofibromas and perineuriomas (1-3). In general, these three tumours can be differentiated depending on their clinical variations, cell composition, growth pattern and immunophenotypic profile. Hybrid tumours of peripheral nerve sheath have been increasingly reported elsewhere, involving more than one histological type, such as schwannoma-neurofibroma, neurofibroma-perineurioma or schwanooma-perineurioma (2-4).

Schwannomas, also known as neurilemomas or neurinomas, are a group of peripheral nerve sheath tumors consisting of Schwann neoplasic cells with variable morphological characteristics (5). Approximately $5 \%$ of the schwannomas appear in the head and neck region, but only $1 \%$ is localised in the oral cavity where tongue is the most frequently affected site, followed by palate, mouth floor, jugal mucosa, lips, gingiva and, more rarely, mandible. Although this tumour may appear at any age, it is more commonly seen in the fourth decade of life $(6,7)$.

In general, schwannomas are asymptomatic encapsulated tumours with slow growth which usually appear in association with a nerve trunk and, as they grow in size, they push the nerve aside. Intra-osseous tumours can cause bone expansion, pain and paresthesia. These lesions are more frequently associated with sensory nerves $(8,9)$.

Perineuriomas are benign neoplasias with advanced perineurial differentiation, being first described in 1978 . These tumours can be classified into two subtypes, namely, intra-neural and extra-neural depending on their clinical and morphological characteristics $(10,11)$. These tumours occur more commonly in the subcutaneous tissues of the trunk and limbs, but can also appear in the dermis and deep soft tissues. Oral perineuriomas have been little reported in the literature, and the clinical-pathological profiles of these neoplasias in the oral cavity have not been well established yet (12,14-17).

Hybrid schwannoma-perineurioma tumours are circumscribed, but they are usually non-encapsulated and present lamellar or storiform architecture similar to a perineurioma. However, they have predominantly a cytomorphology of schwannoma consisting of fusiform cells with wavy and thin nuclei, pale eosinophilic cytoplasm and indistinct cell boundaries $(18,19)$.

The objective of the present study was to present a case of primary intra-osseous tumour with hybrid schwannoma-perineurioma morphology.

\section{Case Report}

A woman attended the Carmino Caricchio Municipal Hospital complaining of diffuse pain in the region of the right mandibular body and of numbness in the right lower lip for about six months. The patient reported that she had undergone extraction of a molar tooth and after the procedure there was a significant worsening of the symptoms. Radiographic examination showed presence of a radiolucent lesion measuring $1.5 \mathrm{~cm}$ in diameter located in the right mandibular body (Fig. 1). The patient

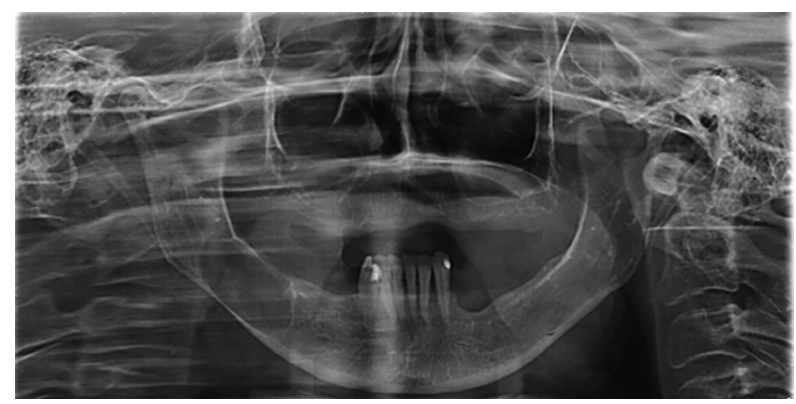

Fig. 1: Panoramic radiography showing radiolucent expansile lesion measuring $1.5 \mathrm{~cm}$ in diameter located in the right mandibular body.

was then submitted to excisional biopsy and the diagnostic hypothesis was a tumour of mesenchymal origin. The biopsy material was sent to Oral and Maxillofacial Surgical Pathology Service, Department of Stomatology, School of Dentistry, University of Sao Paulo. Microscopic analysis revealed fragment of neural tumour, encapsulated, highly cellularised and with fascicular aspect, consisting of fusiform cells with both wavy and hyperchromatic nuclei, or even of epithelioid cells, with scant mitotic activity. Immunohistochemical examination revealed a strong positive labelling for anti S-100 anti-body (Flex Polyclonal Rabbit, anti-S100, readyto-use [Link], DAKO Corporation, Carpinteria, USA) and epithelial membrane antigen (Monoclonal Mouse, anti-human Epithelial Membrane Antigen, clone E29, DAKO Corporation, Carpinteria, USA). CD34 negative immunostaining was observed (Monoclonal Mouse anti-human clone QBEnd-10, Dako Corporation, Carpinteria, USA) (Fig. 2).

After the diagnosis of primary intra-osseous Hybrid Schwannoma-Perineurioma, the patient underwent treatment in the hospital. A panoramic radiography was performed after two years, showing no evidence of relapse of the lesion (Fig. 3).

\section{Discussion}

The majority of the benign peripheral nerve sheath tumours can be classified into a given category depending on their different morphological and immunohistochemical characteristics. In 1998, Feany et al. (2) reported for the first time nine cases of neurofibroma with Schwann cell differentiation. Since then, cases with hybrid characteristics of more than one type of benign peripheral nerve sheath tumour have been successively 

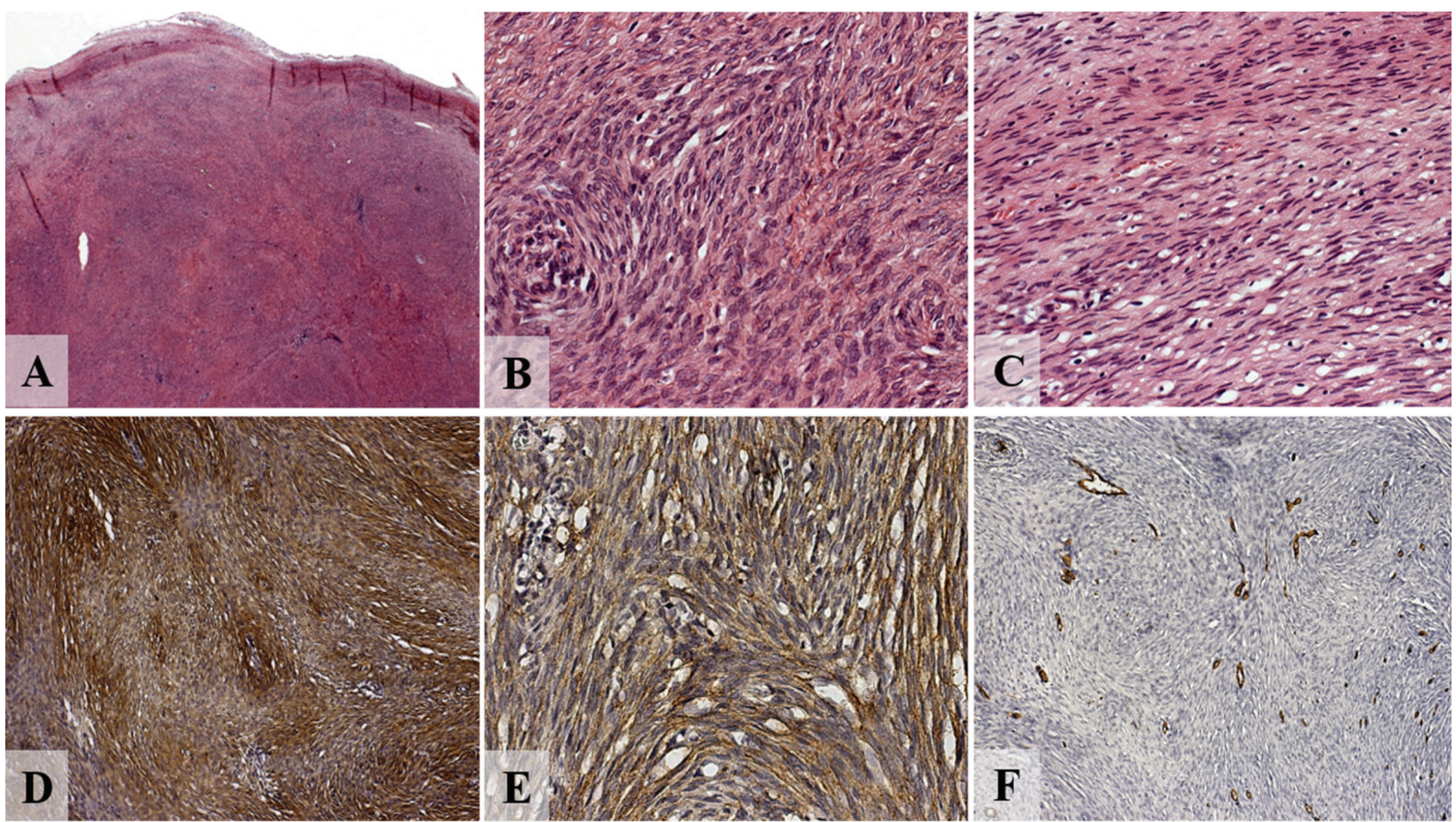

Fig. 2: A) Low power view showing fragment of tumor of neural origin, encapsulated, highly cellularized and of fascicular aspect (hematoxylin and eosin [H\&E], magnification X25); B) this compound being either by fusiform cells, sometimes by cells that exhibit a wavy and hyperchromatic nucleus; C) or even epithelioid cells, with little mitotic activity being noted (H\&E, magnification X400).; D) immunohistochemistry of hybrid neural tumor. The schwannian areas are diffusely positive for S100 (magnification X100); e, epithelial membrane antigen (EMA) is diffusely positive in the spindle cells (magnification X400); f, negative immunostaining for CD34 (magnification X100).

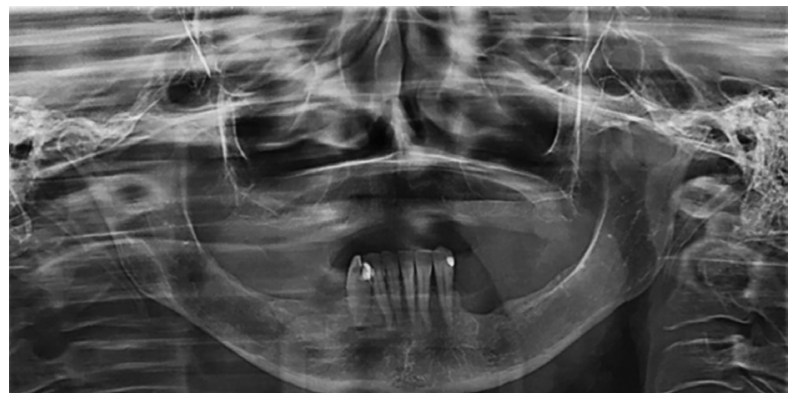

Fig. 3: Panoramic radiography after two years of follow-up showing absence of the lesion.

reported and denominated as hybrid peripheral nerve sheath tumours (HPNST) (19).

Benign peripheral nerve sheath tumours consist of a heterogeneous group of lesions sharing nerve sheath differentiation, but which are clinically, morphologically and immunohistochemically distinct (1). Among them, schwannoma and neurofibroma are the most common histological types, whereas perineurioma is considerably more seldom (20).

These peripheral nerve sheath tumours, in general, are composed of only one histological type. Nevertheless, although tumours with hybrid characteristics of more than one histological type are rare, they have been increasingly more reported in the past years $(13,21)$.
Two forms are represented on the basis of architectonic structures, namely: biphasic tumours (known as neurofibroma-schwannoma or schwannoma-perineurioma), in which two distinct areas are clearly indentified (2-3), and monophasic tumours (known as hybrid schwannoma-perineurioma) $(12,13)$, in which two distinct components are closely intermingled (2-4).

Schwannomas are encapsulated tumors consisting of Schwann cells with a biphasic pattern of dense and loose cellular areas with fusiform nuclei $(22,24)$. Perineuriomas, the rarest lesion in the major triad of peripheral nerve sheath tumours (PNST), are similarly composed of one type of cell only: neoplastic perineurial cell $(12,14-$ 18). Clinically, intraneural perineuriomas have a presentation similar to that of schwannomas, but they can be immunohistochemically distinguished (22-24).

Perineuriomas were positive for EMA and claudin-1, but negative for S100 $(22,23)$. Extra-neural perineuriomas present as extensive soft-tissue masses which can be confirmed by the presence of perineural cell differentiation and perineurial markers claudin-1 and GLUT-1 $(12,14-18)$.

\section{Conclusions}

Benign nerve sheath tumours with predominantly a cytomorphology of schwannoma and perineurioma-type architecture comprise a mixture of both types of cells. 
Histological aspects can suggest a diagnosis, but the use of immunomarkers is needed for confirmation. Despite the limited knowledge on their pathogenesis, these tumours seem to present a non-aggressive biological behaviour. Conservative surgery provides adequate solution without recurrence, even after a long-term follow-up.

\section{References}

1. Yang X, Zeng Y, Wang J. Hybrid Schwannoma/Perineurioma. Report of 10 Chinese Cases Supporting a Distinctive Entity. Int J Surg Pathol. 2013;21:22-8.

2. Feany MB, Anthony DC, Fletcher CD. Nerve sheath tumours with hybrid features of neurofibroma and schwannoma: a conceptual challenge. Histopathol. 1998;32:405-410.

3. Michal M, Kazakov DV, Belousova I, Bisceglia M, Zamecnik M, Mukensnabl P. A benign neoplasm with histopathological features of both schwannoma and retiform perineurioma (benign schwannoma/ perineurioma: a report of six cases of a distinctive soft tissue tumor with a predilection for the fingers. Virchows Arch. 2004;445:347-353. 4. Kazakov DV, Pitha J, Sima R, Vanecek T, Shelekhova K, Mukensnabl P, Michal M. Hybrid peripheral nerve sheath tumors: schwannoma/perineurioma and neurofibroma/perineurioma. A report of three cases in extradigital locations. Ann Diagn Pathol. 2005;9:16-23.

5. Zainab H, Kale AD, Hallikerimath S. Intraosseous schwannoma of the mandible. J Oral Maxillofac Pathol. 2012;16:294-296.

6. Bansal R, Trivedi P, Patel S. Schwannoma of the tongue. Oral Oncol. 2005;41:15-17.

7. Hashiba Y, Nozaki S, Yoshizawa K, Noguchi N, Nakagawa K, Yamamoto E. Recurrent multinodular neurilemmoma of the female upper lip. Int. J. Oral Maxillofac. Surg. 2007;36:171-173.

8. Pandarakalam C, Sudha S, Shameena PM, Varghese VI. An unusual presentation of a case of schwannoma. J Oral Maxillofac Pathol. 2005;9:27-9.

9. da Silva LFM, Duarte BG, Boiça BA, Rocha-Junior HV, Pereira-Stabile CL. Intraoral schwannoma: a case report. Oral Maxillofac Surg. 2013;17:319-321.

10. Lazarus SS, Trombetta LD. Ultrastructural Identification of a Benign Perineurial Cell Tumor. Cancer. 1978;41:1823-1829.

11. da Silva WG, Martínez MM, Miranda AMMA, Silva RB, da Silveira HM, de Almeida OP, et al. Oral Perineurioma: Clinicopathologic Features From Two Cases and Review of Literature. Oral Surg Oral Med Oral Pathol Oral Radiol. 2017;123:e91-e98.

12. Hornick JL, Fletcher CD. Soft tissue perineurioma: clinicopathologic analysis of 81 cases including those with atypical histologic features. Am J Surg Pathol. 2005;29:845-858.

13. Emanuel P, Pertsemlidis DS, Gordon R, Xu R. Benign hybrid perineurioma-schwannoma in the colon. A case report. Ann Diagn Pathol. 2006;10:367-370.

14. Noonan VL, Greene DJ, Brodsky G, Kabani SP. Extraneural sclerosing perineurioma of the buccal mucosa: a case report and clinicopathologic review. Head Neck Pathol. 2010;4:169-173.

15. Siponen M, Sándor GK, Ylikontiola L, Salo T, Tuominen H. Multiple orofacial intraneural perineuriomas in a patient with hemifacial hyperplasia. Oral Surg Oral Med Oral Pathol Oral Radiol Endod. 2007; 104:e38-e44.

16. Boyanton BL Jr, Jones JK, Shenaq SM, Hicks MJ, Bhattacharjee MB. Intraneural perineurioma: a systematic review with illustrative cases. Arch Pathol Lab Med. 2007;131:1382-1392.

17. Adachi S, Mitani K, Iwamoto Y, Furumoto A, Yamashita M, Cho H. Atypical soft tissue perineurioma in the tongue of a Young girl. Pathol Int. 2010;60:787-791.

18. González-Arriagada WA, Leon JE, Vargas PA, Almeida OP, Lopes MA. Intraoral sclerosing perineurioma: a case report and review of the literature. Oral Surg Oral Med Oral Pathol Oral Radiol Endod. 2010;109:e46-e52.
19. Din NU, Ahmad Z, Abdul-Ghafar J, Ahmed R. Hybrid peripheral nerve sheath tumors: report of five cases and detailed review of literature. BMC Cancer. 2017;17: 349.

20. Phan DC, Gleason BC. Recent developments in benign peripheral nerve sheath tumors. J Cutan Pathol. 2008;35:1165-1169.

21. Hornick JL, Bundock EA, Fletcher CDM. Hybrid schwannoma/ perineurioma. Clinicopathologic analysis of 42 distinctive benign nerve sheath tumor. Am J Surg Pathol. 2009;33:1554-1561.

22. Skovronsky DM, Oberholtzer JC. Pathologic classification of peripheral nerve tumors. Neurosurg Clin N Am. 2004;15:157.

23. Lang Y, Liu D, Xiang P, Wang J, Li Y. Primary intraosseous hybrid epithelioid schwannoma/perineurioma in the proximal tibia: a case report of benign hybrid neoplasm with local hypercellularity. Diagn Pathol. 2019;14:1-8.

24. McLaughlin CT, Kaffenberger BH, Gru AA. A Hybrid Tumor With Schwannoma-Perineurioma-Neurofibroma Morphology. J Cutan Pathol. 2015;42:911-3.

\section{Consent for publication}

The patient gave consent for publication of this case report.

\section{Ethical approval}

All procedures performed in studies involving human participants were in accordance with the ethical standards of the institutional and/ or national research committee and with the 1964 Helsinki declaration and its later amendments or comparable ethical standards.

\section{Informed consent}

Informed consent was obtained from all individual participants included in the study.

\section{Conflict of Interest}

The authors declare that they have no conflict interests. 